\title{
Spontaneous Fibrinolysis in Pulmonary Embolism
}

\author{
James E. Wilson III, Eugene P. Frenkel, Alan K. Pierce, Robert L. \\ Johnson, Jr., Edward R. Winga, George C. Curry, and \\ Donald S. Mierzwiak \\ From the Pauline and Adolph Weinberger Laboratory for Cardioptilmonary \\ Research, Department of Internal Medicine, University of Texas (Southwestern) \\ Medical School at Dallas, Dallas, Texas 75235
}

\begin{abstract}
A в S T R A C T This study correlated levels of activated fibrinolysis with the presence, extent, and rate of resolution of angiographically documented pulmonary emboli. Pulmonary emboli demonstrable by angiography were associated with detectable fibrin split products in the serum of 24 of 25 patients. In the absence of increased fibrin split products, pulmonary emboli large enough to be demonstrated by angiography were found in only 2 of 25 positive pulmonary angiograms. Spontaneous resolution of pulmonary emboli could not be correlated with the the concentration or persistence of fibrin split products but did correlate well with the presence of a reversible precipitating cause.

Thrombophlebitis in the absence of clinical evidence of pulmonary embolism was not associated with increased concentrations of fibrin split products in eight of nine patients. The one patient with increased fibrin split product concentration had evidence on lung scan of silent pulmonary embolism.
\end{abstract}

\section{INTRODUCTION}

Spontaneous resolution of acute pulmonary emboli has been demonstrated by direct and indirect techniques. Ventilatory function and gas exchange have been shown to be normal in survivors of pulmonary emboli 1-6 months after the acute insult unless pulmonary emboli were recurrent $(2-4)$. Utilizing serial pulmonary angiograms to assess the extent of resolution of emboli, Fred, Axelrad, Lewis, and Alexander (5) found complete resolution within 2-4 wk in four of five anticoagulated patients with previously normal heart and lungs; three of the four patients with complete resolution had an identi-

This study was presented in part at the 1969 meeting of the American Thoracic Society, Miami, Fla. (1).

Dr. Wilson was a U. S. Public Health Service Special Research Fellow (HE 35,818).

Received for publication 17 June 1970 and in revised form 14 September 1970. fiable, reversible precipitating cause. By contrast, Dalen et al. (6) found complete angiographic resolution in only 2 of 10 patients who were clinically similar to those of Fred et al., except that the presence or absence of a reversible precipitating cause was not stated. Serial lung scans also have suggested variability in the resolution of pulmonary emboli $(7,8)$.

Some of the factors which affect the course of resolution are known. Spontaneous resolution of pulmonary emboli is less complete in patients with very large emboli, preexisting cardiovascular disease (7), or previous venous thromboembolic disease (1). An additional variable is suggested by the data of Genton and Wolf (9), who showed that pulmonary emboli caused by old as compared with fresh thrombi were lysed less rapidly in dogs treated with urokinase.

Fibrinolysis is the final common pathway for resolution of emboli, and some index of fibrinolytic activity might provide criteria for assessing the likelihood of resolution. Furthermore, the level of fibrinolytic activity might prove useful in the diagnosis of pulmonary embolism. The most direct index of fibrinolytic activity is the presence of fibrin split products in serum (10).

The purpose of the present study was to correlate levels of activated fibrinolysis as identified by fibrin split product concentrations, with the presence, extent, and rate of resolution of angiographically proven pulmonary emboli. Fibrin split products were also determined in other thrombotic diseases.

\section{METHODS}

Study plan. Fibrin split product determinations were carried out in normal subjects and in patients with deep thrombophlebitis. The minimal clinical criteria for thrombophlebitis were compatible clinical symptoms, calf tenderness, and at least $1.5 \mathrm{~cm}$ enlargement in circumference of an extremity. In patients with suspected pulmonary emboli, serial fibrin split product concentrations were determined daily for 4 days and then weekly for $4 \mathrm{wk}$; complete clinical 
evaluation, routine laboratory studies, and pulmonary angiograms were carried out in these patients.

Blood collection. Before September 1968, blood samples collected in the absence of venous stasis were immediately clotted with excess thrombin and were incubated for $2 \mathrm{hr}$ at $37^{\circ} \mathrm{C}$. The serum was then separated after centrifugation at $1000 \mathrm{~g}$ for $10 \mathrm{~min}$ and was frozen at $-20^{\circ} \mathrm{C}$ in multiple aliquots for later analysis.

After September 1968, blood was collected in citrate tubes ( $4.5 \mathrm{ml}$ of blood with $0.5 \mathrm{ml}$ of $3.8 \%$ sodium citrate) with $0.1 \mathrm{ml}$ trasylol added. Plasma was separated after centrifugation at $1000 \mathrm{~g}$ and was frozen in multiple $1 \mathrm{ml}$ aliquots at $-20^{\circ} \mathrm{C}$. The day the sample was analyzed, excess thrombin $(100 \mathrm{U} / \mathrm{ml})$ was added to the plasma aliquot, and it was incubated for $2 \mathrm{hr}$ at $37^{\circ} \mathrm{C}$ before the serum was separated for assay.

Evaluation of fibrin split products. Fibrin split products were determined by the hemagglutination-inhibition method of Merskey, Kleiner, and Johnson (10). Hyland antifibrinogen was used after absorption three to five times with lyophilized normal human serum. Sensitivity was calibrated each day with a standard normal plasma. Before September 5,1968 , the lower limit of sensitivity for inhibition of agglutination was $7.5 \mu \mathrm{g}$ of fibrinogen per $\mathrm{ml}$. During this period of time, samples were analyzed from 12 patients with positive angiograms, 5 patients with negative angiograms (2 of whom showed $7.5 \mu \mathrm{g} / \mathrm{ml}$ of fibrin split products), 1 patient with thrombophlebitis, and 10 normal controls. After September 5, 1968, the lowest concentration of standard normal plasma that would inhibit agglutination in the assay was $3.75 \mu \mathrm{g} / \mathrm{ml}$ on 20 occasions and $1.88 \mu \mathrm{g} / \mathrm{ml}$ on 5 occasions. The increased sensitivity was associated with the use of a new lot of Hyland antifibrinogen after September 5, 1968.

Fibrin split product determinations were very reproducible. Aliquots of the same sample were run in duplicate; the results were identical in nine instances, within one tube dilution in four instances, and within two tube dilutions in one instance.

Evaluation of pulmonary angiograms. Pulmonary angiograms were performed during right heart catheterization; they were considered positive only if definite filling defects or cutoffs could be demonstrated (11). The per cent vascular bed occluded was estimated by the following criteria: (a) There are 10 segmental-sized pulmonary artery branches to each lung, each constituting $5 \%$ of the pulmonary vascular bed. (b) The degree of occlusion of a vessel was estimated utilizing the following schema: nearly complete occlusion or a cutoff equals $90 \%$ occlusion; moderate filling, $70 \%$ occlusion; the smallest filling defect discernible, $50 \%$ occlusion. Thus, a moderate filling defect of one segmental vessel equals $0.7 \times 0.05 \times 100=3.5 \%$ occlusion of the total pulmonary vascular bed. (c) If definite filling defects or cutoffs were found, the following were accepted as $50 \%$ occlusion of the segmental vessels involved: (i) beading, (ii) pruning, (iii) arterial hang-ups of contrast material, $(i v)$ poor capillary filling phase, and $(v)$ delayed venous phase.

Evaluation of lung scans. Lung scans performed with ${ }^{181} \mathrm{I}-$ labeled macroaggregated albumin were considered "diagnostic of pulmonary emboli" when perfusion defects were present in the absence of $(a)$ infiltrates in that area on chest roentgenograms, (b) pleural effusion, (c) chronic obstructive pulmonary disease, and $(d)$ bullae.

Lung scan defects were roughly quantitated by the following criteria. Each lung was divided into upper, middle, and lower zones. The smallest perfusion defect that appeared

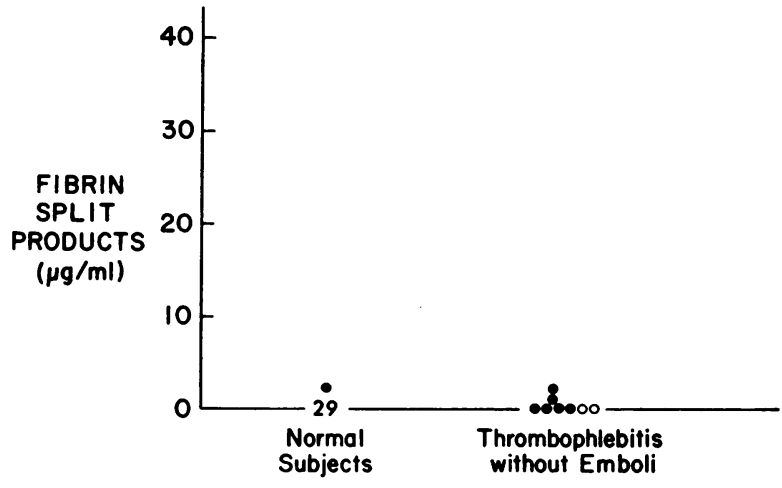

FIGURE 1 Fibrin split products in normal subjects and patients with thrombophlebitis without evidence of emboli. The open circles represent superficial thrombophlebitis.

definite was designated $1+$, an intermediate-sized defect $2+$, and a severe defect $3+$. The grades from each lung zone were then summed; the maximal possible grade was $18+$. A perfusion defect was recorded for an area of radiographic infiltrate if a concurrent pulmonary angiogram demonstrated a definite filling defect. No attempt was made to evaluate a perfusion defect on lung scan in the presence of a pleural effusion.

\section{RESULTS}

Normal subjects and thrombotic states without clinical evidence of pulmonary emboli

29 of 30 clinically normal subjects had no fibrin split products detectable by our method, and one had $3.8 \mu \mathrm{g} /$ ml (Fig. 1). Four patients with uncomplicated deep thrombophlebitis had no demonstrable fibrin split products; one such patient had $3.8 \mu \mathrm{g} / \mathrm{ml}$, and one had 1.9 $\mu \mathrm{g} / \mathrm{ml}$. Two patients with superficial thrombophlebitis had no detectable fibrin split products.

In four patients fibrin split products were zero before and after inferior vena cavagrams. Eight patients had arteriograms for a variety of clinical states, and fibrin split product concentration increased after the procedure in three. In all three instances, the initial value was zero and rose to $3.8 \mu \mathrm{g} / \mathrm{ml}$ in two cases and to $1.9 \mu \mathrm{g} / \mathrm{ml}$ in one case. In all three the fibrin split product concentration was back to zero by the 2nd day after the procedure.

Patients suspected of having pulmonary emboli

Because of the possibility that the catheterization procedure itself might have caused some thrombosis with subsequent lysis, the highest fibrin split product level recorded before the angiogram was placed in Table I as well as Figs. 2 and 3 except for patient L.W. whose first blood sample was $2 \mathrm{hr}$ after the angiogram.

Relationship of fibrin split products to documentation of an embolus. 33 patients suspected of having pulmonary emboli had pulmonary angiograms of diagnostic 
quality. 25 of the 33 had positive angiograms. Fibrin split products were detectable in the serum of all but one of those with positive angiograms (mean 15.2, range
0-30 $\mu \mathrm{g} / \mathrm{ml}$ ) (Fig. 2). In that patient with no detectable fibrin split products only $6 \%$ of the pulmonary vascular bed was occluded, and the first blood sample was ob-

TABLE I

Clinical and Laboratory Features in Those Patients with Pulmonary Angiographic Evidence of Thromboemboli

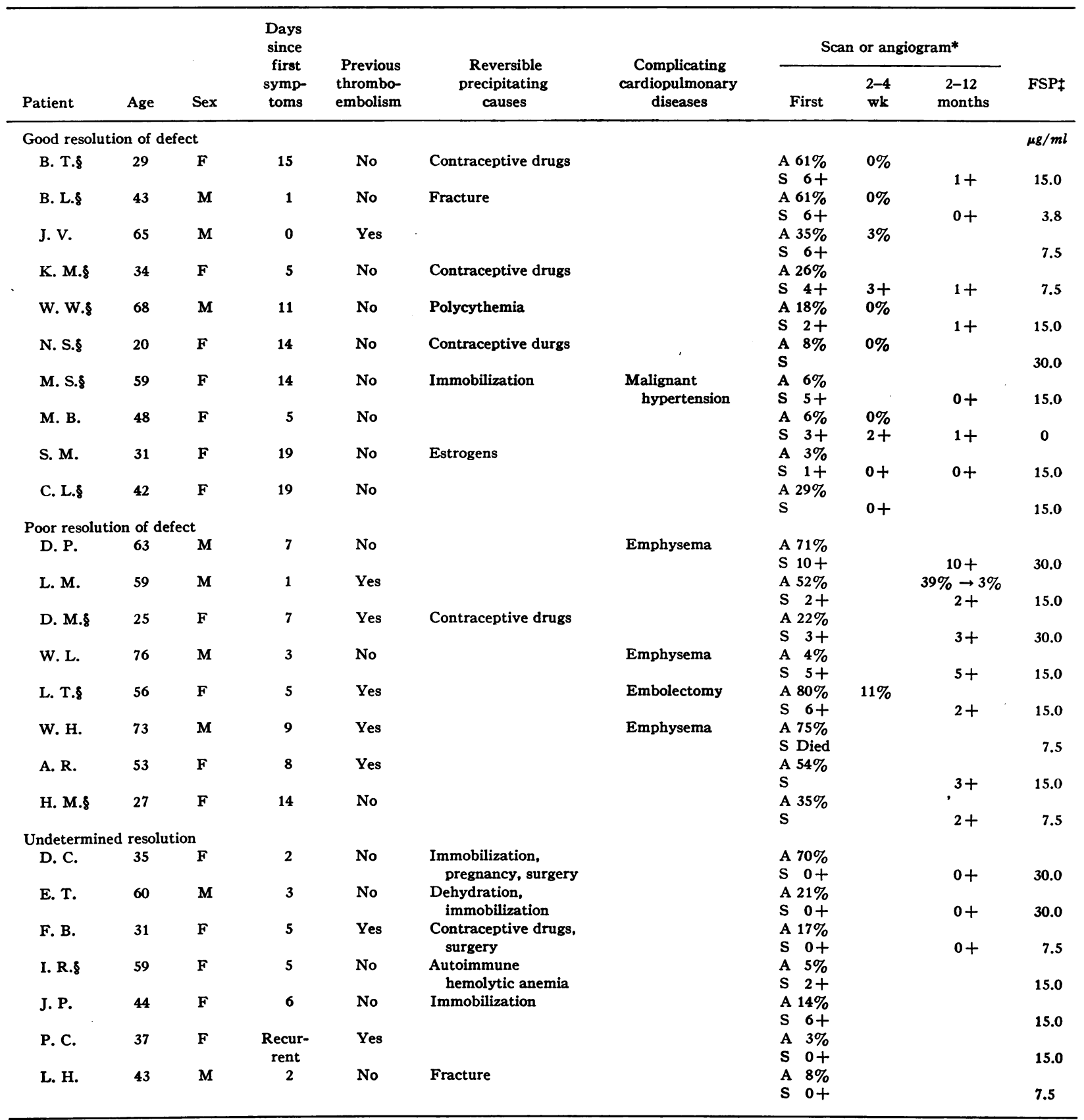

* Estimate of the extent of vascular occlusion by angiography (A) indicated as a per cent occlusion and by scans (S) indicated by a grading system in which $1+$ is the smallest and $18+$ the largest discernible defect (see Methods).

$\mp$ Fibrin split products in serum.

Patients classified as having pulmonary infarction on the basis of a pulmonary infiltrate in the region of the embolic occlusion lasting for longer than

7 days. 
tained 5 days after the probable time of embolism. There was no significant difference $(P>0.4)$ between fibrin split product concentrations in patients with pulmonary infarction (indicated by $\S$ in Table I) and those with emboli but no infarction. Six of the eight patients who had negative pulmonary angiograms had no fibrin split products detectable in their serum, and two had 7.5 $\mu \mathrm{g} / \mathrm{ml}$.

Relationship of fibrin split products to size of vascular bed occluded. In patients demonstrating emboli of a wide range of sizes, there was no correlation between the per cent vascular bed occluded and the concentration of fibrin split products (Fig. 3).

Resolution of emboli. On the basis of combined serial angiograms and lung scans, the patients are represented in Table $\mathrm{I}$ in the following three groups: $(a)$ those who showed good resolution, $(b)$ those with poor resolution, and $(c)$ those with insufficient data to assess resolution of the emboli initially present. Correlations of the concentration of the fibrin split products with the degree of resolution demonstrated that split product values did not predict which patients would markedly clear their pulmonary emboli.

Fig. 4 demonstrates the correlation of the extent of embolism estimated from lung scans with the per cent occlusion estimated from the angiograms. The correlation, although significant $(P<0.01)$, is at times very poor in individual patients. An extreme example is patient D.C. who had $70 \%$ occlusion estimated from her pulmonary angiogram, yet had a normal lung scan.

\section{DISCUSSION}

Diagnostic criteria. Both pulmonary angiograms and lung scans have a significant incidence of false negative interpretations. Moser et al. (11) correlated lung scans and angiograms with autopsy findings in dogs embolized with autologous thrombi. Lung scans missed large emboli more frequently, and angiograms more often missed small emboli; scans missed $17 \%$ of small emboli and $31 \%$ of massive emboli. The two procedures, therefore,

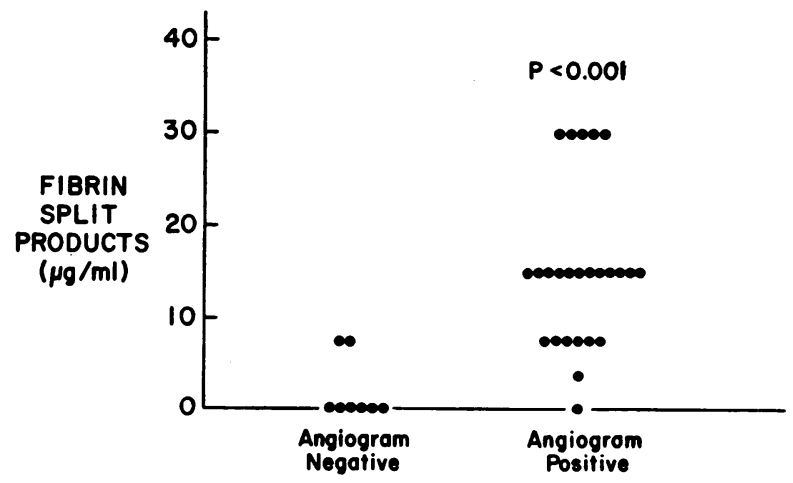

FIGURE 2 Fibrin split product concentrations in patients thought clinically to have pulmonary emboli.

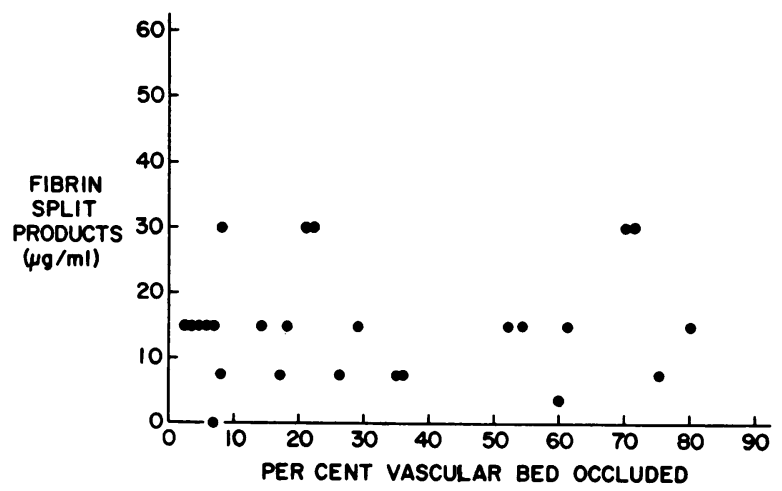

FIGURE 3 Fibrin split product concentrations related to extent of embolization estimated from pulmonary angiograms.

should be complementary in terms of a maximal diagnostic yield.

Moser et al. (11) also found that for small emboli both procedures had frequent false positive interpretations (26\% for lung scans and $28 \%$ for angiograms). However, there was never a false positive interpretation of a filling defect on pulmonary angiograms, and cutoffs were the next most reliable criteria. In the present study, 21 of the patients diagnosed as emboli had filling defects on angiograms, and another 4 had cutoffs. Two of the patients with cutoffs had diagnostic lung scans with perfusion defects in regions of no infiltrate. The other two patients had repeat pulmonary angiograms showing complete revascularization of the previously cutoff vessels. Our reliance on such strict interpretation of pulmonary angiograms, eliminating

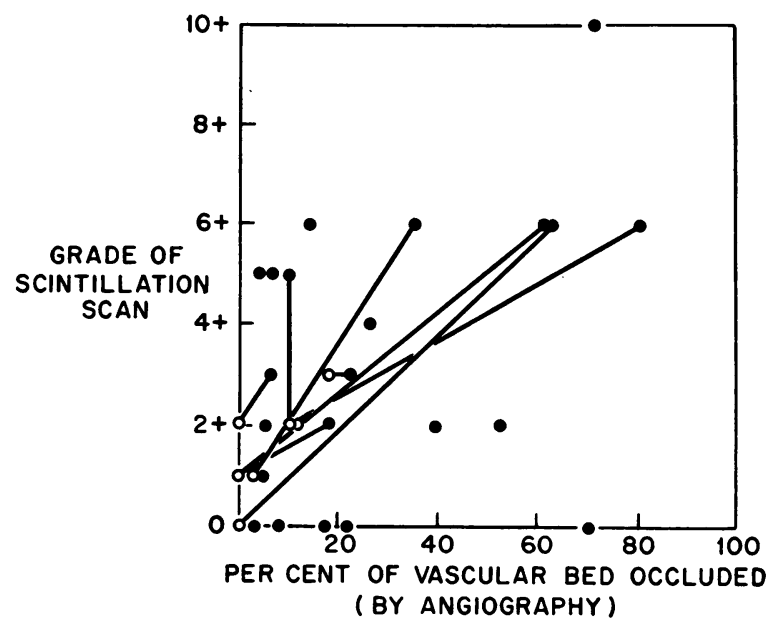

FIGURE 4 Correlation between the extent of embolization estimated by lung scans and angiograms. The closed circles represent the results of the initial lung scan compared with the corresponding angiogram. When both studies were repeated, the repeat comparisons are designated by an open circle and are connected by a straight line to the corresponding first study. $\mathrm{n}=30, r=0.505, P<0.01$. 
patients with positive scans but negative angiograms, may have caused underdiagnosis of multiple small emboli; however, we wished to reduce false positive diagnoses to a minimum. 8 of the 25 patients with positive angiograms had less than $10 \%$ of the pulmonary vascular bed occluded, a situation in which it is difficult to be absolutely certain about an angiographic defect. However, 5 of the these patients had confirmation by disappearance of the defect on a repeat angiogram and/ or a diagnostic lung scan. The criteria, for estimating the extent of occlusion were arbitrary. For instance, a filling defect arbitrarily was considered to reflect $50 \%$ occlusion of the vessel even though hemodynamically the effect of the partially obstructing clot might be considerably more or less than this estimate. Furthermore, major filling defects on an angiogram representing clots partially occluding the main pulmonary arteries might not be evident at all on a lung scan if the increased resistance to flow is evenly distributed between the two lungs. Some defects are hard to pick up either on a scan or an angiogram without serial follow-up procedures which enable the observer to note the disappearance of a previously existing but questionable abnormality. This may account for the better correlations in those instances where the scans and angiograms were repeated (closed and open circles connected by straight lines in Fig. 4).

Methods of studying fibrinolytic activity. Patients with pulmonary emboli diagnosed clinically without pulmonary angiograms or lung scans have had their potential for fibrinolysis studied by indirect in vitro methods. Tsitouris, Sandberg, DeLeon, Lecks, and Bellet (12) reported increased inhibitors of fibrinolysis by an antiplasmin lysis time in 11 of 14 patients. The method used did not distinguish between antiactivators and antiplasmins; however, activator inhibitors are very rare (13). Ellison and Brown (14) found a 50\% whole blood clot lysis time normal in thromboembolic pulmonary hypertension, whereas 9 of 11 patients with a history of pulmonary infarction had a prolonged $50 \%$ clot lysis time, which suggests poor spontaneous fibrinolysis in the latter group. However, these methods of evaluation may not be reflective of the in vivo occurrence of fibrinolysis.

When a thrombus forms, plasminogen is incorporated within the fibrin meshwork (15). Plasminogen activators from either circulating plasma or vascular intima (16) diffuse into the thrombus and convert plasminogen to plasmin which lyses fibrin; systemic hyperplasminemia may not be present during active fibrinolysis (17). Intima from human veins and the adventitial layer of arteries and veins have marked fibrinolytic activity, but the imtima of human arteries is low in such activity (18-23). The intima of pulmonary arteries is similar to veins and has good fibrinolytic activity (20). If a small amount of plasminogen is converted to plasmin within the systemic circulation, antiplasmins complex with the plasmin and prevent degradation of fibrinogen. When the complex contacts fibrin, the plasmin is released owing to the plasmin's greater affinity for fibrin than for antiplasmin (24). The plasmin digests fibrin and releases fibrin split ploducts which can be detected in the serum by immunologic techniques. Fibrin split products have the same antigenic sites as fibrinogen, but are not clottable by thrombin. These fibrin split products provide the best evidence available that fibrinolysis has occurred (10); thus, we chose to study what we considered the best assessment of active fibrinolysis in vivo.

There is adequate evidence from other sources that fibrin split products are detectable in the serum after thromboembolism. Merskey et al. (10) found fibrin split products in the serum of five patients with pulmonary emboli. Laursen and Gormsen (25) found only four of seven patients with pulmonary emboli to have fibrin split products; however, emboli were diagnosed clinically without pulmonary angiograms. They found fibrin split products in only 2 of 15 patients with phlebothrombosis, and one of these patients had a complicating pulmonary embolus. Niléhn and Nilsson (26) found fibrin split products in five of six patients with thrombosis; it is not clear whether the thrombi were arterial or venous. Their method also detected fibrin split products in the serum of 5 of 28 normal individuals.

Predicting resolution of emboli. Some factors that might predict the resolution of a pulmonary embolus are: (a) fibrinolytic activity $(12,14),(b)$ size of the embolus, (7), (c) preexisting cardiovascular disease (7), (d) recurrent thromboembolic disease (2-4), and (e) existence of a reversible precipitating cause (5). Fibrin split product concentration was not predictive of good resolution, and its simultaneous assessment weighted by the per cent vascular bed occluded was not helpful. Serial fibrin split product concentrations are listed in Table II. The duration of increased fibrin split products varied from 2 days to over 30 days after heparin therapy was started and also was not helpful in predicting resolution. Two patients, who both had $61 \%$ occlusion of their pulmonary vascular bed, had fibrin split products detectable in their serum for only 2 and 3 days yet had complete resolution of their filling defects on repeat pulmonary angiograms. The only factor that would have significantly predicted good resolution was the presence of a reversible precipitating cause $(P<0.05)$. A history of previous thromboembolism was present in a majority of those with poor resolution and was rare in those with good resolution, but the differences were not significant.

Near the end of the study it became apparent that fibrin split product concentrations could not predict good spontaneous resolution of emboli, and several patients 
were studied with the limited objective of testing the correlation of elevated fibrin split products and a positive pulmonary angiogram.

Screening test for pulmonary emboli. In the present study, when the serum concentration of fibrin split prod- ucts in patients with proven emboli is compared with the concentrations in patients with negative pulmonary angiograms (Fig. 2), the association of increased concentrations of fibrin split products above $5 \mu \mathrm{g} / \mathrm{ml}$ with a positive angiogram is highly significant $(P<0.001)$

TABLE II

Serial Fibrin Split Product Determinations

\begin{tabular}{|c|c|c|c|c|c|c|c|c|}
\hline \multirow[b]{3}{*}{ Patient } & \multirow{3}{*}{$\begin{array}{c}\text { Interval } \\
\text { between } \\
\text { first symptom } \\
\text { and entrance } \\
\text { into study }\end{array}$} & \multicolumn{7}{|c|}{ Interval after entrance into study (days) } \\
\hline & & \multicolumn{2}{|c|}{$1-3$} & \multirow[b]{2}{*}{ 4-7 } & \multirow[b]{2}{*}{ 8-14 } & \multirow[b]{2}{*}{$15-21$} & \multirow[b]{2}{*}{$22-28$} & \multirow[b]{2}{*}{ 29-90 } \\
\hline & & $\begin{array}{c}\text { Before } \\
\text { angiogram }\end{array}$ & $\begin{array}{c}\text { After } \\
\text { angiogram }\end{array}$ & & & & & \\
\hline \multicolumn{9}{|c|}{ days } \\
\hline \multicolumn{9}{|c|}{ Fibrin split product concentrations $(\mu g / m l)$ in patients with good resolution } \\
\hline B. $\mathrm{T}$. & 14 & $15, \downarrow 15$ & & 0,0 & 0 & & & 0 \\
\hline B. L. & 5 & $3.8 \downarrow$ & $0,1.9$ & 0 & $\mathbf{0}$ & & 0 & \\
\hline J. V. & 0 & $7.5 \mathrm{~V} \downarrow$ & $120,7.5$ & & 30 & & & $7.5,7.5$ \\
\hline K. M. & 5 & $7.5, \downarrow 7.5$ & 7.5 & & 15 & & 0 & \\
\hline W. W. & 11 & $\downarrow 15$ & 60 & $15,7.5$ & & & $15,7.5$ & \\
\hline N. S. & 14 & $\downarrow 30$ & 15 & 30 & 15,15 & 0 & 0 & 0 \\
\hline M.S. & 13 & 15 & & & & & & \\
\hline M. B. & 4 & $0, \downarrow 0$ & 0 & 0 & 0,0 & 0,0 & & \\
\hline S. M. & 19 & 15 & $\downarrow 15$ & 15 & & & 7.5 & 0,0 \\
\hline C. L. & 18 & $15, \downarrow 15$ & 7.5 & $7.5,0,0$ & $15 \mathrm{~V}$ & & & \\
\hline \multicolumn{9}{|c|}{ Fibrin split product concentration $(\mu \mathrm{g} / \mathrm{ml})$ in patients with poor resolution } \\
\hline D. P. & 6 & $\downarrow 3.8,30$ & & 15 & & & & \\
\hline L. M. & 1 & $\downarrow 15$ & 15,60 & 15 & $15 \mathrm{~V}$ & & & 0 \\
\hline D. $\mathbf{M}$. & 4 & $30, \downarrow 30,30$ & & $7.5,7.5$ & 0 & $3.8,0$ & $\mathbf{0}$ & \\
\hline W. L. & 3 & $15,15 \downarrow$ & & & $7.5,7.5$ & 3.8 & & \\
\hline L. T. & 3 & $\downarrow 15,15$ & & E60 & $\mathrm{T}$ & 60 & 30,30 & $* 7.5,7.5,0,0,0$ \\
\hline W. H. & 9 & 7.5 & $\mathrm{~V} 15,15,15$ & Expired & & & & \\
\hline A. $\mathbf{R}$. & 8 & $\downarrow 15$ & 15 & & & & & \\
\hline H. Mc. & 14 & 7.5 & $\downarrow$ & & & & & \\
\hline
\end{tabular}

Fibrin split product concentration $(\mu \mathrm{g} / \mathrm{ml})$ in patients with negative pulmonary angiograms
N. H.
$\downarrow 0$
E. C.
$0 \downarrow$
0
0,0
$\mathbf{0}$
$\mathbf{0}$
0
$0 \quad 0$
$0,0,7.5$
M. H.
V. H.
M. P.
L. S.
L. W.
0, $\downarrow 10,0$
$0, \downarrow 0$
$\downarrow 0,0$
Recurrent
\begin{tabular}{lcccc} 
L. W. & Recurrent & \multicolumn{3}{c}{$7.5,15,15$} \\
D. H. & 3 & $\downarrow 7.5$ & 0 & $7.5,0$
\end{tabular}

$0 \quad 3.75$
0
$7.5,15,15$

Fibrin split product concentration $(\mu \mathrm{g} / \mathrm{ml})$ in patients with thrombophlebitis

$\begin{array}{lrccc}\text { J. J. } & 58 & \downarrow 0 & 0 & \\ \text { E. J. } & 8 & 0 \downarrow & 0 & \\ \text { J. C. } & 15 & \downarrow 0,0,0 & & \\ \text { J. L. } & 3 & \downarrow 0,0 & & \\ \text { R. T. } & 5 & \downarrow 1.9,0,0 & & \\ \text { E. C. } & 15 & 60 & 0 & 0 \\ \text { B. A. } & 2 & \downarrow 3.8,3.8 & 0 & 0 \\ \text { A. K. } & 7 & \downarrow 0,0 & & \\ \text { L. W. } & 14 & 0 & \end{array}$

$\downarrow=$ onset of heparin therapy; $\mathrm{V}=$ vena cava ligation; $\mathrm{T}=$ new episode of thrombophlebitis; $\mathrm{E}=$ embolectomy.

* Value at 4-6 months.

† Superficial thrombophlebitis. 
by chi-square analysis. Thus, in the absence of fibrin split products a patient is not likely to have had an embolus large enough to detect with pulmonary angiography. These data suggest that the fibrin split product determination could be a useful screening test for emboli large enough to be detected by pulmonary angiograms. Fibrin split products were increased as late as 3 wk after the embolic episode; however, in some patients on heparin therapy the split products disappeared as rapidly as 2 days after the embolic episode. The effect of heparin therapy upon the duration of detectable fibrin split products in the serum is not known but needs to be investigated.

Although the absence of fibrin split products in the serum may be good suggestive evidence that an embolus large enough to be detected by angiography has not occurred, the existence of fibrin split products in serum does not necessarily indicate thromboembolism. The incidence of fibrin split products in the serum of patients with nonembolic pulmonary disease is still not known but probably is significant. It is of interest that in uncomplicated deep thrombophlebitis and in two cases of superficial thrombophlebitis there was no increase in fibrin split products in the patient's sera. One patient not listed in Fig. 1 appeared to be an exception, since the concentration of fibrin split products was $60 \mu \mathrm{g} / \mathrm{ml}$ in the presence of deep thrombophlebitis. However, a lung scan demonstrated the occurrence of asymptomatic pulmonary emboli.

\section{ACKNOWLEDGMENTS}

We are indebted to Dr. Clarance Merskey of the Albert Einstein College of Medicine, New York, and to his technicians, Santosh Magon and Joy Lawrence, for their advice about fibrin split product determinations.

This work was supported by grants from the U. S. Public Health Service (HE 06296 and GM 14892) and by a grant from the Dallas Heart Association. Dr. Winga was a Trainee supported by U. S. Public Health Service Training Grant HE 05812.

\section{REFERENCES}

1. Wilson, J. E., III, E. R. Winga, E. P. Frenkel, A. K. Pierce, and R. L. Johnson, Jr. 1969. Evidence of spontaneous fibrinolysis in thrombophlebitis and pulmonary embolism. Amer. Rev. Resp. Dis. 99: 974. (Abstr.)

2. Emanuel, D. A., R. D. Sautter, and F. J. Wenzel. 1966. Conservative treatment of massive pulmonary embolism. J. Amer. Med. Ass. 197 : 924.

3. Colp, C. R., and M. H. Williams, Jr. 1962. Pulmonary function following pulmonary embolization. Amer. Rev. Resp. Dis. 85: 799.

4. Wilson, J. E., III, A. K. Pierce, R. L. Johnson, Jr., E. R. Winga, W. R. Harrell, G. C. Curry, and C. B. Mullins. 1971. Hypoxemia in pulmonary embolism, a clinical study. J. Clin. Invest. 50: 481.

5. Fred, H. L., M. A. Axelrad, J. M. Lewis, and J. K. Alexander. 1966. Rapid resolution of pulmonary throm- boemboli in man: an angiographic study. $J$.Amer. Med. Ass. 196: 1137.

6. Dalen, J. E., J. S. Banas, Jr., H. L. Brooks, G. L. Evans, J. A. Paraskos, and L. Dexter. 1969. Resolution rate of acute pulmonary embolism in man. N. Engl. J. Med. 280: 1194.

7. Tow, D. E., and H. N. Wagner, Jr. 1967. Recovery of pulmonary arterial blood flow in patients with pulmonary embolism. N. Engl. J. Med. 276: 1053.

8. Murphy, M. L., and R. T. Bulloch. 1967. The resolution of pulmonary emboli as determined by angiography and scanning. Clin. Res. 15: 348. (Abstr.)

9. Genton, E., and P. S. Wolf. 1967. Experimental pulmonary embolism: effects of urokinase therapy on organizing thrombi. J. Lab. Clin. Med. 70: 311.

10. Merskey, C., G. J. Kleiner, and A. J. Johnson. 1966. Quantitative estimation of split products of fibrinogen in human serum, relation to diagnosis and treatment. Blood. 28: 1 .

11. Moser, K. M., P. Harsanyi, G. Rius-Garriga, M. Guisan, G. A. Landis, and A. Miale, Jr. 1969. Assessment of pulmonary photoscanning and angiography in experimental pulmonary embolism. Circulation. 39: 663.

12. Tsitouris, G., H. Sandberg, A. C. DeLeon, Jr., L. Lecks, and S. Bellet. 1960. Studies of the plasmin-plasminogen system in thromboembolic diseases: its modifications by thrombolysin therapy. Amer. J. Cardiol. 5: 680.

13. Nilsson, I. M., H. Krook, N.-H. Sternby, E. Söderberg, and N. Söderström. 1961. Severe thrombotic disease in a young man with bone marrow and skeletal changes and with a high content of an inhibitor in the fibrinolytic system. Acta Med. Scand. 169: 323.

14. Ellison, R. C., and J. Brown. 1965. Fibrinolysis in pulmonary vascular disease. Lancet. 1: 786.

15. Sherry, S. 1968. Fibrinolysis. Annu. Rev. Med. 19: 247.

16. Kwaan, H. C., and T. Astrup. 1965 Fibrinolytic activity in thrombosed veins. Circ. Res. 17: 477.

17. Alkjaersig, N., A. P. Fletcher, and S. Sherry. 1959. The mechanism of clot dissolution by plasmin. J. Clin. Invest. 38: 1086.

18. Astrup, T. 1966. The relation between formation and lysis of fibrin in the body. Thromb. Diath. Haemorrh. Suppl. 20: 71.

19. Lieberman, J., and F. Kellogg. 1961. Fibrinolytic activity of arterial tissues. Circ. Res. 9: 515.

20. Todd, A. S. 1959. The histological localisation of fibrinolysin activator. J. Pathol. Bacteriol. 78: 281.

21. Astrup, T. 1966. Tissue activators of plasminogen. Fed. Proc. $25: 42$.

22. Pandolfi, M., I. M. Nilsson, B. Robertson, and S. Isacson. 1967. Fibrinolytic activity of human veins. Lancet. 2: 127.

23. Coccheri, S., and T. Astrup. 1961. Thromboplastic and fibrinolytic activities of large human vessels. Proc. Soc. Exp. Biol. Med. 108: 369.

24. Ambrus, C. M., and G. Markus. 1960. Plasmin-antiplasmin complex as a reservoir of fibrinolytic enzyme. Amer. J. Physiol. 199: 491.

25. Laursen, B., and J. Gormsen. 1967. Spontaneous fibrinolysis demonstrated by immunological technique. Thromb. Diath. Haemorrh. 17: 42.

26. Niléhn, J.-E., and I. M. Nilsson. 1964. Demonstration of fibrinolytic split products in human serum by an immunological method in spontaneous and induced fibrinolytic states. Scand. J. Haematol. 1: 313. 